\title{
Lymphomatoid contact dermatitis caused by limonene hydroperoxides confirmed by an exposure provocation test with the involved personal hygiene products.
}

María-Elena Gatica-Ortega,(1) María-Antonia Pastor-Nieto,(2)

Cristina Schoendorff-Ortega, (1) Manuela Mollejo-Villanueva,,(3)

Ana Giménez-Arnau (4)

Dermatology (1) and Pathology (3) Depts.Toledo Hospital Complex (Toledo, Spain)

Dermatology Dept. University Hospital of Guadalajara (Guadalajara, Spain). Alcalá de Henares University. (Alcalá de Henares, Madrid) (2) Department of Dermatology, Hospital del Mar. Institut Mar d'Investigacions Médiques, Universitat Autònoma. (Barcelona, Spain) (4).

\section{Correspondence}

María Elena Gatica-Ortega

Carretera de Cobisa s/n cp:45071

Dermatology Department

Toledo Hospital Complex (Toledo, Spain)

Complejo Hospitalario de Toledo. Toledo (España).

mail: mariaelenagatica@yahoo.com.ar

Authors do not have any conflicts of interests to declare. 


\section{KEY WORDS}

Allergic contact dermatitis; lymphomatoid contact dermatitis; limonene hydroperoxides; fragrances; pseudolymphoma; cutaneous T cell lymphoma; mycosis fungoides; limonene; lymphomatoid; cosmetics. 


\section{INTRODUCTION}

Lymphomatoid contact dermatitis is an uncommon inflammatory disorder of the skin which is classified as a pseudolymphoma. It is likely an under-reported entity, with clinical and histological features of cutaneous T cell lymphoma (CTCL) while showing some characteristics of allergic contact dermatitis. Etiologically, it has been linked to several haptens such as phosphorus sesquisulfide (1), gold, nickel, formalin, dimethyl fumarate, methylisotiazolinone and azo dyes. Contact allergen hypersensitivity is presumed to be the key in its pathogenesis, allergen avoidance usually leads to the resolution of the process and clonality cannot be proved in most cases.

The first case of lymphomatoid contact dermatitis in relation to limonene hydroperoxides is reported.

\section{CASE REPORT}

A 35-year-old non-atopic female was referred to us with a pruritic eruption that had started four months earlier. Her family history revealed a diagnosis of parapsoriasis affecting her mother. Lesions, consisting of erythematous wellcircumscribed plaques with thin scales, initially involved her lower abdomen to eventually spread to her lower limbs (Figure 1a). The eruption partially responded to a methylprednisolone aceponate ointment. According to the clinical features and her family history, an early onset of a CTCL was suspected.

Cutaneous pathology showed a perivascular and perifollicular slightly atypical lymphoid infiltrate with focal epidermotropism (Fig. 1 b). Immunostaining highlighting the presence of CD4+Tcells within the epidermis (Fig. 1c). 
However, T-cell receptor gene (TCR) rearrangements could not be identified by polymerase chain reaction tests (PCR).

The patient was patch tested with the baseline Spanish Contact Dermatitis Research Group (GEIDAC) series with TrueTest ${ }^{\circledR}$ and supplementary allergens supplied by AllergEaze (Calgary, Canada), cosmetic series (Chemotechnique, Vellinge, Sweden) and five of her own hygiene products. The allergens were prepared on Curatest ${ }^{\circledR}$ chambers (Lohmann \&Rauscher, Neuwied, Germany), applied on the patient's back and fixed with an adhesive tape (Omnifix $\mathrm{E}^{\circledR}$, Hartmann, Heidenheim, Germany). Exposure times and readings were performed and scored according to the European Society of Contact Dermatitis (ESCD) guidelines (2).

Positive results were obtained with limonene hydroperoxides (LimOOHs) $0.3 \%$ pet., 1+ on day 4 (D4), and with one of her own products ("The hair pack"), 1+ on D2 and D4 (Figure 1d). The patient claimed to suffer from severe pruritic papules when she used it.

Limonene was labeled in several of the patient's hygiene and household products such as the hair pack, as well as her shower gel, moisturizing cream, shampoo, washing-up liquid and floor mop.

Topical corticosteroids and sun baths ( $5^{\prime} /$ day) were recommended and the patient was given limonene avoidance instructions.

These measures led to a complete clearance of the lesions in a few months. After strictly avoiding contact with limonene and being completely asymptomatic for 15 months, a cutaneous provocation test was accepted by the patient with an informed consent. She started using her former shampoo, shower gel and a 
moisturizing cream containing limonene for one month. A flare up of lesions similar to the previous ones appeared over the same previously affected abdominal locations (Figure $2 \mathrm{a}$ ).

The patient was patch tested a second time with Lim-OOHs $0.3 \%$ and linalool hydroperoxides $1 \%$. Positive results were obtained with Lim-OOHs $0.3 \%$ pet. (1+) on D2, D4 (Figure 2 b). Patch tests with limonene 10\% pet.

(Chemotechnique, Vellinge, Sweden) were negative on D2, D4 and D7. A new biopsy revealed focal parakeratosis and lymphoid dermal infiltrates with focal epidermotropism (Fig. 2 c) of mostly CD3+CD8+ lymphocytes (Fig. 2 d) with isolated eosinophils. Polyclonal TCR gene rearrangements were identified by PCR tests

Topical corticosteroids and limonene avoidance resulted in a fast improvement.

\section{DISCUSSION}

Limonene is a common fragrance terpene with a fresh citrus odor widely used in cosmetic, household and hygiene industries. It has recently been recognized as a pre-hapten meaning a substance prone to be turned into more allergenic compounds through air oxidation. The limonene Lim-OOHs have been described to be important contact haptens. According to a recent study, the prevalence of Lim-OOHs sensitization within the overall patch tested population in Spain is $5.1 \%$ and Lim-OOHs $0.3 \%$ pet. have been proposed to be a useful screening test (3). To the best of our knowledge, no other cases of lymphomatoid contact dermatitis from Lim-OOHs have been previously reported thus far. 
Lymphomatoid contact dermatitis was originally described in 1976 by GómezOrbaneja et al. (1) involving four patients who developed cutaneous lesions from the matchboxes carried in their pockets. Biopsies were consistent with CTCL and the culprit allergen was found to be the phosphorus sesquisulfide in the matchboxes. Lesions recovered after avoiding further contact from it.

In 2014, a literature review was published by Knackstedt and Zug (4). Among 23 cases, lymphomatoid contact dermatitis was more frequent in male patients (median age: 58.5). Fourteen different haptens were identified. Most frequent locations were thighs, head and neck, buttocks and groin. Most cases (68.1\%) showed strong (2+) or extremely strong (3+) patch test reactions and only $31.9 \%$ had week reactions (1+). In most cases (80\%) recovery was achieved with topical steroids and allergen avoidance. Airborne -induced lymphomatoid contact dermatitis from methylisothiazolinone has also been reported (5).

In accordance with the literature, our patient improved after avoiding the allergen. Unlike previous reports, our case involved a female-patient showing more generalized lesions and patch test reactions were weaker (1+).

We believe that allergen re-exposure tests could be the way to diagnose sensitization from ubiquitous allergens such as fragrances like $\mathrm{Lim}-\mathrm{OOHs}$ especially when, as in our case, patch test reactions are not strong. In these cases, clinical relevance is difficult to determine and complete allergen avoidance hard to accomplish.

We believe that it is very important to consider the diagnosis of lymphomatoid contact dermatitis in lesions resembling early-onset mycosis fungoides, especially in those cases with pruritic lesions, atypical body distribution and 
polyclonal infiltrates, and recommend patch tests are performed with a broad allergen pannel. 


\section{AKNOWLEDGEMENTS}

To the patient for kindly collaborating with our investigation. To Kevin Brown for his unselfish assistance with the English correction of the paper. 


\section{REFERENCES}

1. Orbaneja J G, Diez L I, Lozano J L, Salazar LC. Lymphomatoid contact dermatitis: a syndrome produced by epicutaneous hypersensitivity with clinical features and histopathologic picture similar to that of mycosis fungoides. Contact Dermatitis 1976: 2: 139-143.

2. Johansen JD, Aalto-Korte K, Agner T, et al. European Society of Contact Dermatitis guideline for diagnostic patch testing -recommendations on best practice. Contact Dermatitis 2015: 73: 195-221.

3. Deza G, García-Bravo B, Silvestre JF, et al. Contact sensitization to limonene and linalool hydroperoxides in Spain: a GEIDAC* prospective study. Contact Dermatitis. 2017: 76: 74-80.

4. Knackstedt TJ, Zug KA. T cell lymphomatoid contact dermatitis: a challenging case and review of the literature. Contact Dermatitis 2014: 72: $65-74$.

5. Van Steenkiste E, Goossens A, Meert H, et al. Airborne -induced lymphomatoid contact dermatitis caused by methylisothiazolinone. Contact Dermatitis 2015: 72: 237-240. 


\section{FIGURES}

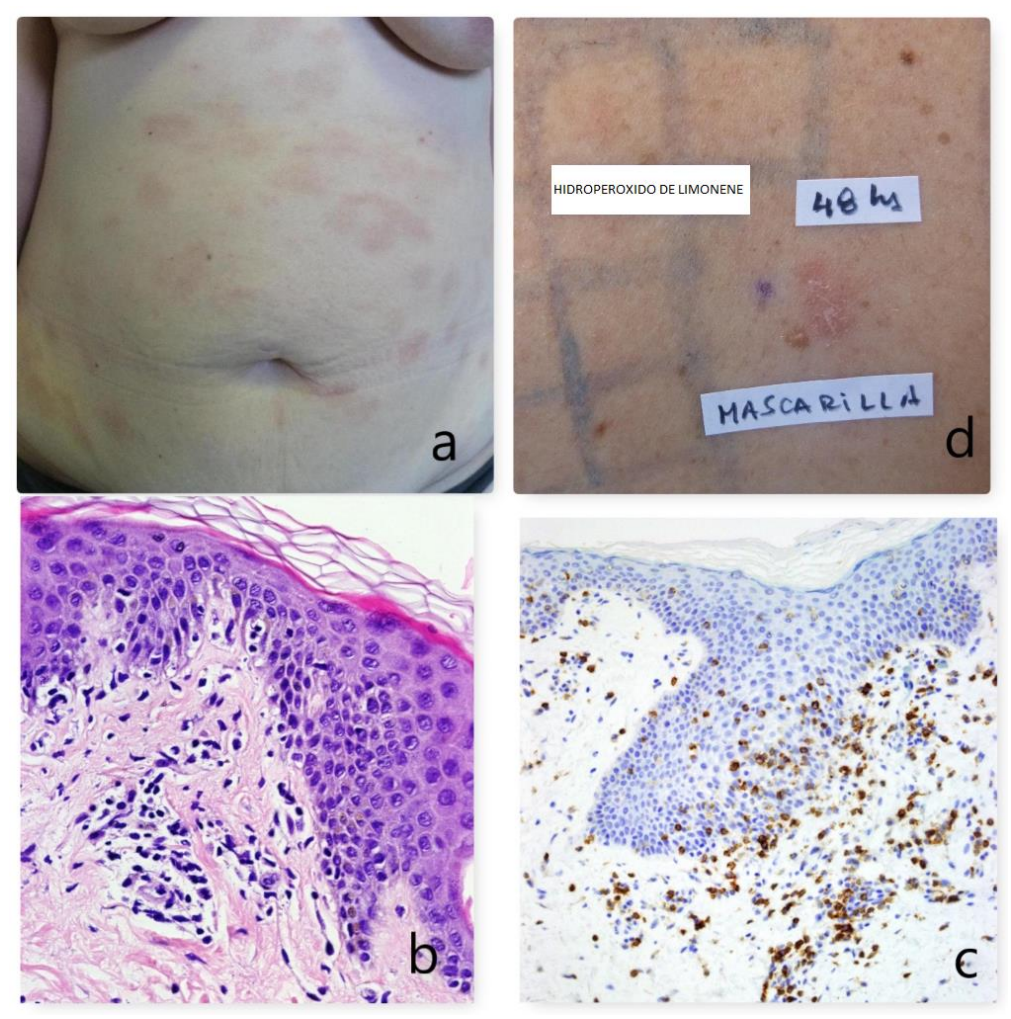

FIGURE 1: (a) Numerous erythematous, well-delimited, maculopapular lesions scattered over the abdomen. (b) Histopathological examination showing a slight epidermotropism of atypical lymphocytes and minimal spongiosis without eosinophils. (Haematoxylin and eosin H\&E x 400); (c) Immunostaining highlighting the presence of CD4+Tcells within the epidermis (x 200). (d) Patch test results: limonene hydroperoxides $0.3 \%$ pet., ? on day 2 (D2); patient's own hair mask product ("The hair pack") $1+$ on D2. 

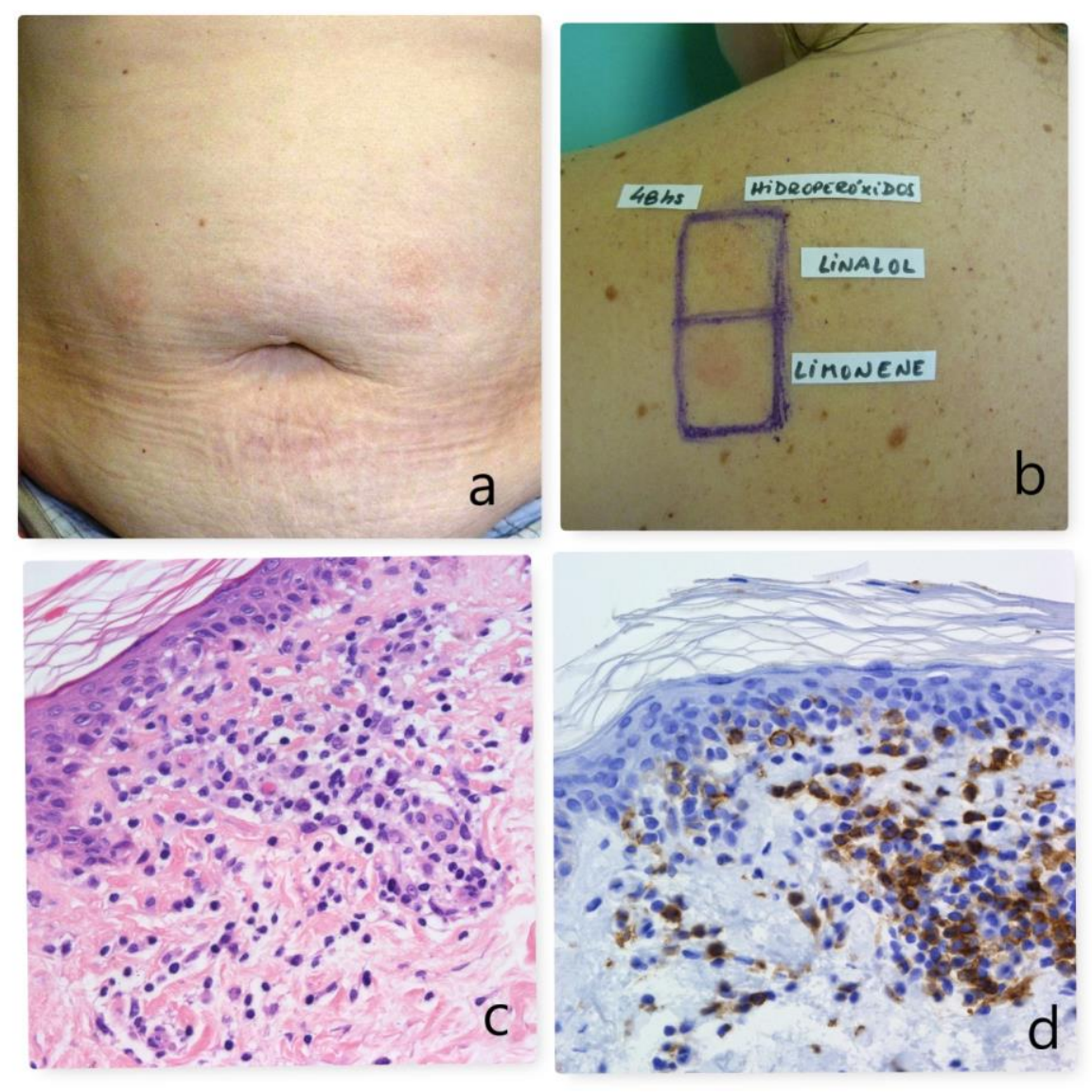

FIGURE 2: (a) Scarce erythematous, well delimited, macular lesions involving the abdomen. (b) Patch test results: limonene hydroperoxides $0.3 \%$ pet., $1+$ on day 2 (D2); (c) High power view revealing limited epidermotropism with atypical lymphocytes and minimal spongiosis (H\&E, x400). (d) Immunohistochemistry highlighting the presence of CD8+ Tcells within the epidermis ( $x 400)$. 